\title{
Dosimetric Comparison of Simultaneous Integrated vs. Sequential Boost in Radiotherapy for High Grade Gliomas
}

\author{
Tahani Nageeti*, Mohamed Mahfouz, Heba Abdallah, Mazen Algaoud and Reem Zatar \\ Department of Radiation Oncology, King Abdullah Medical City -Holy Capital, Saudi Arabia
}

Submission: October 01, 2019; Published: November 07, 2019

"Correspondence Author: Tahani Nageeti, Consultant Radiation Oncology, 2677 Almasheer District, Makkah, Saudi Arabia

\begin{abstract}
Objective: To compare the dosimetric coverage of the Planning target volumes (PTV) and sparing organs at risk (OARs) in volumetric modulated arc therapy (VMAT) technique for high grade glioma using different methods to Boost the tumor bed by sequential boost (SEB) vs. simultaneous integrated boost (SIB).

Methods: Non-interventional dosimetric study of seven consecuti7e cases with pathologically proven high-grade gliomas who attended KAMC radiotherapy were included in the study. We delineated PTVs and the boost volumes (BVs) on the planning CTs with image fusion with MRIs. Delineation of the target volumes was based on the gross target volume (GTV) which is defined as the contrast enhancing visible tumor on the T1 with gadolinium (Gd) images. The clinical target volume (CTV1), representing the subclinical tumor involvement, is defined as GTV1 +15.0 -mm expansion including the edema visible on the T2-weighted images. The planning target volume (PTV1) is defined as CTV1 + 5.0-mm margin. The CTV2 is defined as the GTV + 5.0-mm expansion including the contrast-enhancing tumor visible on the T1-Gd images. The PTV2 is defined as the CTV2 $+5.0 \mathrm{~mm}$ margin. We planned each case by three VMAT plans of the (SB) technique and two (SIB) techniques of two dose regimens utilizing equivalent biologically effective doses for all plans. The first Plan using of the SB, PTV1 received 46 Gy over 23 fractions in 2 Gy as dose per fraction and PTV2 received 14 Gy in seven fractions with 2Gy as dose per fraction. The second Plan) using SIB planning (SIB1) , the PTV1 received 54 Gy over 30 fractions with 1.8 Gy as a dose per fraction, while the PTV2 received 60 Gy over 30 fractions but with 2 Gy as a dose per fraction. The third plan was carried out using SIB (SIB2) with different biologically equivalent dose to deliver to PTV-1 dose of 48.6 Gy over 27 fractions in 1.8 Gy as dose per fraction while the PTV-2 will receive 59.4 Gy over 27 fractions but with 2.2 Gy as a dose per fraction.
\end{abstract}

Results: We compared the dose distributions and DVH for OAR constrains for all three plans. We found that the mean percentage coverage of $95 \%$ of PTV1 volume $(109 \%, 96.49 \%$ and $96.23 \&)$ for (SB, SIB1 and SIB2) respectively. The mean percentage coverage of $95 \%$ of PTV2 volume were $(98 \%, 96.8 \%$ and $94.26 \%$ ) for (SB, SIB1 and SIB2) respectively. The mean maximum dose to optic nerve is (19.7 Gy, $20 \mathrm{~Gy}, 18.23 \mathrm{GY}$ ) for (SB, SIB1 and SIB2) respectively which is statistically non-significate with (P-value 0.94). The mean maximum dose to optic chiasm ( $40.3 \mathrm{GY}$, $41.76 \mathrm{GY}$, $38 \mathrm{GY}$ ) for (SB, SIB1 and SIB2) respectively which is also statistically non-significate with P-value $<0.9$. We found no statistical difference into the mean maximum dose to brainstem (55.6 GY, 54 GY, 50.2 GY) for (SB, SIB1 and SIB2), respectively (P-value= 0.057).

Conclusion: We found no significant difference in the mean maximum dose to organs at risk in utilizing VMAT for planning SB vs SIB in highgrade glioma. Based on our data we recommend that utilization of SIB with a smaller number of fractions of biologically equivalent to standard fractionation might be the best option for treatment of high-grade glioma patients.

Keywords: Brain Radiotherapy; High Grade Gliomas; VMAT; Simultaneous Integrated Boost; Sequential Boost

\section{Introduction}

Despite of the use of combined treatment modalities including surgery, radiotherapy, and chemotherapy for the management of high-grade gliomas, the prognosis is still poor with a median survival of 14.6 months following combined treatment [1]. These tumours exhibit an infiltrative growth and it is necessary to control the peripheral infiltrating parts of microscopic tumor, which is usually not visible on imaging, as well as the visible tumor on diagnostic imaging. Therefore, it very important to target both areas with deliver different dose levels trying to spare the critical structures while maximize the dose to the area at risk [2-7]. Intensity modulated radiotherapy (IMRT) is advantageous owing to its better dose distribution and better sparing of normal tissues when compared with traditional 3D radiation treatment [8-11]. Volumetric-modulated arc therapy (VMAT) is a special kind of rotational IMRT [12]. In VMAT during rotation of the gantry, the dose rate and shape 


\section{Cancer Therapy \& Oncology International Journal}

of multileaf collimator were dynamically modulated. One of the advantages of IMRT and VMAT is that it is suitable for the application of the simultaneous integrated boost (SIB) method. SIB offers the possibility to apply a dose to the larger volume, that called planning target volume (PTV), and at the same time deliver an escalated dose to the smaller target at tumor mass, that called gross tumor volume (GTV) $[2,13,14]$.

According to ICRU-83, the GTV represents the palpable or visible (on imaging) tumor, whereas the clinical target volume (CTV) is an additional volume with a certain probability of microscopic (subclinical) malignant disease. The irradiated PTV is a geometrical concept, which comprises the CTV plus a safety margin [15]. This safety margin compensates for the effects of organ and patient movement during the radiation fraction and for inaccuracies in patient set-up. Of course, in brain tumors, organ movement is of minor importance. In other words, it was shown that this technique provided improved target conformity and better sparing of OARs. Because of its superior spatial resolution, magnetic resonance imaging (MRI) is favoured in the management of high-grade gliomas $[2,15]$.

It is a well-known fact that higher dose per fraction is potentially more toxic regarding the development of late radiation side effects such as necrosis of central nervous system tissues and other injuries [16-18]. Thus, in addition to "physical sparing" of OARs, the simultaneous integrated boost (SIB) method can also offer the possibility of a "biological sparing" as in SIB method, the single dose per fraction to the PTV not boost tumor volume (BTV) was lower when compared with the SIB. That might be of interest in patients with brain tumors with longer life expectancies and when considering that in the PTV there is a comparatively high probability of irradiation of subvolumes of relatively sensitive OARs [19]. The Primary Objective of the study was to compare the dosimetric coverage of the Planning target volumes (PTV) and sparing the critical structures and organs at risk (OARs) using different delivery methods of radiation to Boost Tumor volume (BTV) for high grade Glioma, using sequential boost (SEB) vs. simultaneous integrated boost (SIB) using VMAT. The Secondary Objective was to assess other factors might suggest the suitability of different methods: Tumor location, presence of peri-tumor edema, presence of ring enhancement on MRI, and clinical target volume CTV (cc).

\section{Methods}

The study design was non-interventional dosimetric study, which included cases for patients treated with radiotherapy for pathologically proven high-grade gliomas that underwent either total or subtotal surgical resection of their tumors between Jan and March 2017 and received radiation treatment based on multidisciplinary decision at King Abdulla Medical City (KAMC). Ethical approval was obtained from King Abdulla Medical CityIRB (KAMC IRB). Since this was a dosimetric non-interventional study patient consents were waived. We included all cases with diagnosed and pathologically proven high-grade gliomas (WHO Grade III, IV gliomas, anaplastic astrocytoma AA, anaplastic oligo-astrocytoma AOA and anaplastic oligo-dendroglioma AO). All cases were treated with partial brain irradiation and with radical intent. The cases that were treated with whole brain irradiation or with palliative intent or low-grade histology were excluded from the study.

We delineated PTVs and the BVs on the planning CTs with image fusion with MRIs. Delineation of the target volumes was based on the gross target volume (GTV) which is defined as the contrast enhancing visible tumor on the $\mathrm{T} 1$ with gadolinium (Gd) images. The clinical target volume (CTV1), representing the subclinical tumor involvement, is defined as GTV1 + 15.0$\mathrm{mm}$ expansion including the edema visible on the T2-weighted images. The planning target volume (PTV1) is defined as CTV1 $+5.0-\mathrm{mm}$ margin. The CTV2 is defined as the GTV + 5.0-mm expansion including the contrast-enhancing tumor visible on the T1-Gd images. The PTV2 is defined as the CTV2 $+5.0 \mathrm{~mm}$ margin. The study was focus on comparing the dose distributions in the isodose plans and DVH of the sequential boost (SEB) technique and two simultaneous integrated boost (SIB) method techniques in two dose regimens both by volumetric modulated arc therapy (VMAT).

All patients had CT- Simulation with thermoplastic head mask, Intravenous contrast and with $2 \mathrm{~mm}$, CT-san slides thickness. All the contoured volumes transferred to the Eclipse system using DICOM. Treatment planning and calculation of the dose distributions for both methods (SIB and SEB) carried out for all patients in the Eclipse planning system. Treatment planning for both methods were done using Arc/VMAT technique. Three different plans will be done for each case. The first Plan (P1) was conducted using of the SEB method, where PTV1 will receive 46 Gy over 23 fractions in 2 Gy as dose per fraction. PTV2 received 14 Gy in 7 fractions with 2 Gy as dose per fraction. The second Plan (P-2) was conducted using SIB planning; the PTV1 I received 54 Gy over 30 fractions with 1.8 Gy as a dose per fraction, while the PTV2 received 60 Gy over 30 fractions but with 2 Gy as a dose per fraction. The third plan (P-3) was conducted using SIB planning with using different biologically equivalent dose to deliver to PTV-1 dose of 48.6 Gy over 27 fractions in 1.8 Gy as dose per fraction while the PTV-2 received 59.4 Gy over 27 fractions but with 2.2 Gy as a dose per fraction (Table 1). Normalization of all plans (SEB and SIB) were done to a mean dose of $100 \%$ of the prescription dose to the target volume. The dose constraints for the OARs were corresponding to their biologically equivalent dose tolerance in $2 \mathrm{~Gy}$ as a dose per fraction: 56 Gy for the brainstem; 54 Gy for the optic nerves, chiasm, retinas, and eyeballs; 40 Gy for the lachrymal glands; and 5 Gy for the lenses. Two independent planners carried out plans for all cases for all three methods in order to achieve the most appropriate coverage of target volumes and sparing of OARs. 


\section{Cancer Therapy \& Oncology International Journal}

Table 1: Biological Effective Dose for different dose Schedule.

\begin{tabular}{|c|c|c|c|c|}
\hline $\begin{array}{c}\text { Total } \\
\text { Dose }\end{array}$ & $\begin{array}{c}\text { Dose/ } \\
\text { Fraction }\end{array}$ & $\begin{array}{c}\text { Number of } \\
\text { Fractions }\end{array}$ & $\begin{array}{c}\text { BED (3) Late } \\
\text { Side Effects }\end{array}$ & $\begin{array}{c}\text { BED (10) } \\
\text { Tumor Control } \\
\text { /Acute Side } \\
\text { Effects }\end{array}$ \\
\hline 60 & 2 & 30 & 100 & 72 \\
\hline 54 & 1.8 & 30 & 86.4 & 63.72 \\
\hline 60 & 2 & 30 & 100 & 72 \\
\hline 48.6 & 1.8 & 27 & 77.8 & 57.35 \\
\hline 59.4 & 2.2 & 27 & 102 & 72.5 \\
\hline
\end{tabular}

\section{Results}

We compared the dose distributions and DVH for OAR constrains for all three plans. We found that the mean percentage coverage of $95 \%$ of PTV1 volume (109\%, 96.49\% and $96.23 \%$ ) for (SB, SIB1 and SIB2), respectively. The mean percentage coverage of $95 \%$ of PTV2 volume were (98\%, 96.8\% and $94.26 \%$ ) for (SEB, SIB1 and SIB2), respectively. The mean maximum dose to optic nerve is (19.7 Gy, 20 Gy, 18.23 GY) for (SEB, SIB1 and SIB2), respectively which is statistically non-significate with a P-value $=0.94$. The mean maximum dose to optic chiasm ( $40.3 \mathrm{GY}, 41.76$ GY, 38 GY) for (SEB, SIB1 and SIB2), respectively which is also statistically non-significate with a P-value $<0.9$. We found no statistical difference into the mean maximum dose to brainstem (55.6 GY, 54 GY, 50.2 GY) for (SEB, SIB1 and SIB2), respectively (P-value $=0.057$. We also found that SIB plans provide less dose spillage to non-target brain tissue when compared to SEB plan.

\section{Discussion}

Recently, VMAT is increasingly utilized in clinical radiation therapy for multiple tumor sites such as prostate cancer, head and neck tumors, and brain tumors. The VMAT and IMRT are advantageous because of its allowed better dose conformality to the target and better sparing of critical structures when compared with traditional 3D radiation therapy. Utilization of these techniques allowed the introduction of the concept of dose escalation for high-grade gliomas. Tsien et al. treated 38 consecutive patients with a dose escalation (66 to 81 Gy over 6 weeks) with concurrent Temozolomide (TMZ), using IMRT for SIB and with a median follow-up of 54 months, the median overall survival was 20 months, and the authors showed that patients could safely undergo 75 Gy in 30 fractions with concomitant TMZ [20]. A retrospective study of 12 patients with high grade glioma treated postoperatively with SIB-IMRT and dose escalation to high risk PTV reach (62.5- 70 Gy) (2.5 Gy per fraction) with concurrent TMZ showed that this approached was tolerable but with high risk of radiation necrosis (RN) [21].

Few comparative studies for SIB vs SEB were carried out in brain tumors. Farzin et al. compared SIB vs SEB in 20 patients with high-grade gliomas. In the SIB method, the PTV received 54 Gy in 30 fractions with 1.8Gy dose per fraction, while the tumor bed received 60 Gy in 2 Gy per fraction [22]. There results demonstrated that the SIB and SEB were equivalent in target coverage, but the SIB was significantly superior to the SEB for almost all the OARs sparing. In our study, we found no statistical difference in the mean maximum dose to optic nerve, optic chiasm and brain stem in all three plans. We used VMAT to plan for both the SIB and the SEB, therefore, it could be expected that the homogeneity and conformity of the doses to the target volumes would be similar for both plans, although the spillage was less in case of SIB plans. Although in our study that OARs sparing was equivalent for all plans, the result suggests that utilization of SIB with a smaller number of fractions of biologically equivalent total dose to standard fractionation might be the best option for treatment of patients with short survival without increasing the toxicity.

\section{Conclusion}

We found that utilizing VMAT for planning SEB vs SIB in highgrade glioma were equivalent in terms of the mean maximum dose to organs at risk. Based on our findings we recommend utilization of SIB with a smaller number of fractions that are biologically equivalent to standard dose for treatment of highgrade glioma patients.

\section{References}

1. Stupp R, Mason WP, van den Bent MJ, Weller M, Fisher B, et al. (2005) Radiotherapy plus concomitant and adjuvant temozolomide for glioblastoma. N Engl J Med 352(10): 987-996.

2. Cho KH, Kim J-Y, Lee SH, Yoo H, Shin SH, et al. (2010) Simultaneous integrated boost intensity modulated radiotherapy in patients with high grade gliomas. Int J Radiat Oncol Biol Phys 78(2): 390-397.

3. Iuchi T, Hatano K, Narita Y, , Kodama T, Yamaki T, et al. (2006) Hypofractionated high dose irradiation for the treatment of malignant astrocytomas using simultaneous integrated boost technique by IMRT. Int J Radiat Oncol Biol Phys 64(5): 1317-1324.

4. Prados MD, Wara WM, Sneed PK, McDermott M, Chang SM, et al. (2001) Phase III trial of accelerated hyperfractionation with or without difluromethylornithine (DFMO) versus standard fractionated radiotherapy with or without DFMO for newly diagnosed patients with glioblastoma multiforme. Int J Radiat Oncol Biol Phys 49(1): 71-77.

5. Nieder C, Andratschke N, Wiedenmann N, Busch R, Grosu AL, et al. (2004) Radiotherapy for high-grade gliomas. Does altered fractionation improve the outcome? Strahlenther Onkol 180(7): 401-407.

6. Selker RG, Shapiro WR, Burger P, Blackwood MS, Arena VC, et al. (2002) The Brain Tumor Cooperative Group NIH Trial 87-01: a randomized comparison of surgery, external radiotherapy, and carmustine versus surgery, interstitial radiotherapy boost, external radiation therapy, and carmustine. Neurosurgery 51(2): 343-355.

7. Souhami L, Seiferheld W, Brachman D, Podgorsak EB, Werner-Wasik M, et al. (2004) Randomized comparison of stereotactic radiosurgery followed by conventional radiotherapy with carmustine to conventional radiotherapy with carmustine for patients with glioblastoma multiforme: report of Radiation Therapy Oncology Group 93-05 protocol. Int J Radiat Oncol Biol Phys 60(3): 853-860.

8. Sveistrup J, af Rosenschöld PM, Deasy JO, Oh JH, Pommer T, et al. (2014) Improvement in toxicity in high risk prostate cancer patients treated with image-guided intensity-modulated radiotherapy compared to 3D conformal radiotherapy without daily image guidance. Radiat Oncol 9: 44. 
9. Mok G, Gauthier I, Jiang H, Huang SH, Chan K, et al. (2015) Outcomes of intensity modulated radiotherapy versus conventional radiotherapy for hypopharyngeal cancer. Head Neck 37(5): 655-661.

10. Matthiesen C, Herman TD, Singh H, Mascia A, Confer M, et al. (2015) Dosimetric and radiobiologic comparison of 3D conformal, IMRT, VMAT and proton therapy for the treatment of early stage glottic cancer. J Med Imaging Radiat Oncol 59(2): 221-228.

11. Burnet NG, Jena R, Burton KE, Tudor GS, Scaife JE, et al. (2014) Clinical and practical considerations for the use of intensity-modulated radiotherapy and image guidance in neuro-oncology. Clin Oncol (R Coll Radiol) 26(7): 395-406

12. Otto K (2008) Volumetric modulated arc therapy: IMRT in a single gantry arc. Med Phys 35(1): 310-317.

13. Suzuki M, Nakamatso K, Kanamori S, Okumra M, Uchiyama T, et al. (2003) Feasibility study of the simultaneous integrated boost (SIB) method for malignant gliomas using intensity-modulated radiotherapy (IMRT). Jpn J Clin Oncol 33(6): 271-277.

14. Thilmann C, Zabel A, Grosser KH, Hoess A, Wannenmacher M, et al. (2001) Intensity-modulated radiotherapy with an integrated boost to the macroscopic tumor volume in the treatment of high-grade gliomas. Int J Cancer 96(6): 341-349.

15. Nakamatsu K, Suzuki M, Nishimura Y, Kanamori S, Koike R, et al. (2008) Treatment outcomes and dose-volume histogram analysis of simultaneous integrated boost method for malignant gliomas using intensitymodulated radiotherapy. Int J Clin Oncol 13(1): 48-53.

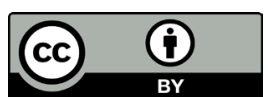

This work is licensed under Creative Commons Attribution 4.0 License DOI: 10.19080/CTOIJ.2019.15.555907
16. Lee AW, Kwong DL, Leung SF, Tung SY, Sze WM, et al. (2002) Factors affecting risk of symptomatic temporal lobe necrosis: significance of fractional dose and treatment time. Int J Radiat Oncol Biol Phys 53(1): 75-85.

17. Marks JE, Baglan RJ, Prassad SC, Blank WF (1981) Cerebral radionecrosis: incidence and risk in relation to dose, time, fractionation and volume. Int J Radiat Oncol Biol Phys 7(2): 243-252.

18. Ruben JD, Dally M, Bailey M, Smith R, McLean CA, et al. (2006) Cerebral radiation necrosis: incidence, outcomes, and risk factors with emphasis on radiation parameters and chemotherapy. Int J Radiat Oncol Biol Phys 65(2): 499-508.

19. Mostafa Farzin, Michael Molls, Sabrina Astner, Rondak IC, Oechsner M, et al. (2015) Simultaneous integrated vs. sequential boost in VMAT radiotherapy of high-grade gliomas. Strahlenther Onkol 191: 945-952.

20. Tsien Christina I, Brown D, Normolle D, Schipper M, Piert M, et al. (2012) Concurrent temozolomide and dose-escalated intensity-modulated radiation therapy in newly diagnosed glioblastoma. Clin Cancer Res18(1): 273-279.

21. Cha J, Suh CO, Park K, Chang JH, Lee KS, et al. (2014) Feasibility and outcomes of hypofractionated simultaneous integrated boost-intensity modulated radiotherapy for malignant gliomas: a preliminary report. Yonsei Med J 55(1): 70-77.

22. Farzin M, Molls M, Astner S, Rondak IC, Oechsner M et al. (2015) Simultaneous integrated vs. sequential boost in VMAT radiotherapy of high-grade gliomas. Strahlentherapie und Onkologie 19(12): 945-952.

\section{Your next submission with Juniper Publishers will reach you the below assets}

- Quality Editorial service

- Swift Peer Review

- Reprints availability

- E-prints Service

- Manuscript Podcast for convenient understanding

- Global attainment for your research

- Manuscript accessibility in different formats

( Pdf, E-pub, Full Text, Audio)

- Unceasing customer service

Track the below URL for one-step submission https://juniperpublishers.com/online-submission.php 